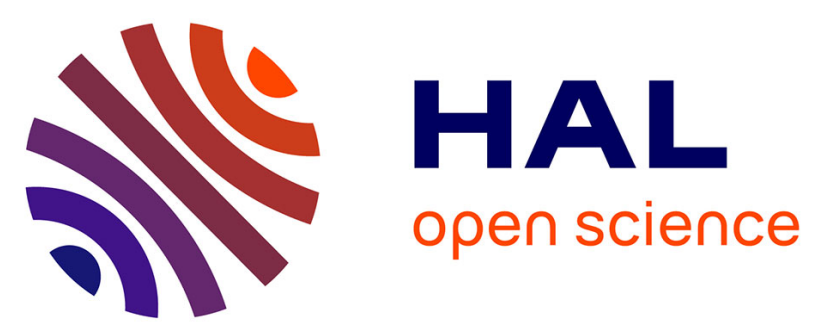

\title{
Monocular 3D Reconstruction and Augmentation of Elastic Surfaces with Self-occlusion Handling
}

Nazim Haouchine, Jeremie Dequidt, Marie-Odile Berger, Stephane Cotin

\section{To cite this version:}

Nazim Haouchine, Jeremie Dequidt, Marie-Odile Berger, Stephane Cotin. Monocular 3D Reconstruction and Augmentation of Elastic Surfaces with Self-occlusion Handling. IEEE Transactions on Visualization and Computer Graphics, 2015, pp.14. 10.1109/TVCG.2015.2452905 . hal-01186011

\section{HAL Id: hal-01186011 \\ https://inria.hal.science/hal-01186011}

Submitted on 23 Aug 2015

HAL is a multi-disciplinary open access archive for the deposit and dissemination of scientific research documents, whether they are published or not. The documents may come from teaching and research institutions in France or abroad, or from public or private research centers.
L'archive ouverte pluridisciplinaire HAL, est destinée au dépôt et à la diffusion de documents scientifiques de niveau recherche, publiés ou non, émanant des établissements d'enseignement et de recherche français ou étrangers, des laboratoires publics ou privés. 


\title{
Monocular 3D Reconstruction and Augmentation of Elastic Surfaces with Self-occlusion Handling
}

\author{
Nazim Haouchine, Jeremie Dequidt, Marie-Odile Berger and Stephane Cotin
}

\begin{abstract}
This paper focuses on the 3D shape recovery and augmented reality on elastic objects with self-occlusions handling, using only single view images. Shape recovery from a monocular video sequence is an underconstrained problem and many approaches have been proposed to enforce constraints and resolve the ambiguities. State-of-the art solutions enforce smoothness or geometric constraints, consider specific deformation properties such as inextensibility or resort to shading constraints. However, few of them can handle properly large elastic deformations. We propose in this paper a real-time method that uses a mechanical model and able to handle highly elastic objects. The problem is formulated as an energy minimization problem accounting for a non-linear elastic model constrained by external image points acquired from a monocular camera. This method prevents us from formulating restrictive assumptions and specific constraint terms in the minimization. In addition, we propose to handle self-occluded regions thanks to the ability of mechanical models to provide appropriate predictions of the shape. Our method is compared to existing techniques with experiments conducted on computer-generated and real data that show the effectiveness of recovering and augmenting 3D elastic objects. Additionally, experiments in the context of minimally invasive liver surgery are also provided and results on deformations with the presence of self-occlusions are exposed.
\end{abstract}

Index Terms_-Image-guided Simulation, Physics-based Modeling, Non-rigid Registration, Computer Assisted Surgery, Elastic Augmented Reality.

\section{INTRODUCTION}

3D recovery and augmentation of deformable objects in a monocular context is a challenging problem with many potential applications in computer graphics, augmented reality and medical imaging. The difficulties originate from the fact that the problem is under-constrained. To overcome this problem, various approaches have been considered with the aim to provide additional constraints and solve the ambiguities. Many approaches introduced deformation models which are often learned from training data and derive models with few degrees of freedom. A lot of papers have been devoted to inelastic materials such as papers, sails, clothes, and make use of the inextensibilty constraint, ensuring that the distance between points remains constant. Other geometric or shading constraints have been proposed to handle materials that can stretch. However, the additional constraints that are used are not always suited to the intrinsic properties of the object. That is the reason why we advocate in this paper the use of a mechanical model within a non-linear elasticity framework to enable reconstruction and augmentation of highly deformable objects. Our model only requires the knowledge of the Young's modulus. Mechanical models have been seldom used in the past due the difficulty to acquire the parameters of the model and the complexity of non-linear models which preclude real time algorithms. In this paper, we propose an efficient real-time algorithm for recovery and augmentation of highly elastic objects in a monocular context. We also show that a rough estimation of the Young's modulus is sufficient to obtain a good reconstruction while imposing sufficient boundary constraints. Applications of the method are provided in the context of augmented reality for minimally invasive liver

- Nazim Haouchine and Stephane Cotin are with INRIA and IHU Strasbourg, France.E-mail: nazim.haouchine@inria.fr, stephane.cotin@inria.fr

- Jeremie Dequidt is with INRIA and Lille University, France.

- Marie-Odile Berger is with INRIA and Lorraine University, France. surgery [1]. Augmented Reality techniques are considered as a well-suited approaches in order to enrich the visual feedback of surgeons during minimally invasive procedures, where surgeons do not directly manipulate the organ but interact with it using instruments inserted through small incisions around the abdominal cavity [2] [3]. Visualization of additional pre-operative information-such as tumors- in the field of view of the surgeon requires to track in real time potential large elastic deformations of the liver. Several techniques that take into account organ elasticity [4], [5], [6], [7] have been proposed. These methods rely on a combination of a stereo estimation of organ motion and on biomechanical models to characterize the elastic behaviour. Despite the fact that these methods yield good results, stereo-laparoscopic cameras are uncommon compared to mono-laparoscopic ones, and this motivated us to investigate this limitation.

The paper is organized as follows: existing techniques for 3D recovery of elastic objects are presented in Section 2. We first review the existing techniques for modeling 3D deformable surfaces and then review non-rigid reconstruction approaches in the monocular context. Our method is detailed in Section 4 and 5. The obtained results are presented in Section 7 and show that the method is able to cope with large elastic deformations in a various dataset including surgery data.

\section{Modeling Non-RIGID OBJects}

The choice of an appropriate 3D deformable model is important for modeling non-rigid objects. In this section we give a brief overview of popular methods that were adopted especially by the computer vision community, namely physically-based models, mass-spring systems, learned models and parametric models. They have been proven to be suitable for applications related (but not limited) to face tracking, medical registration and non-rigid shape recovery. 

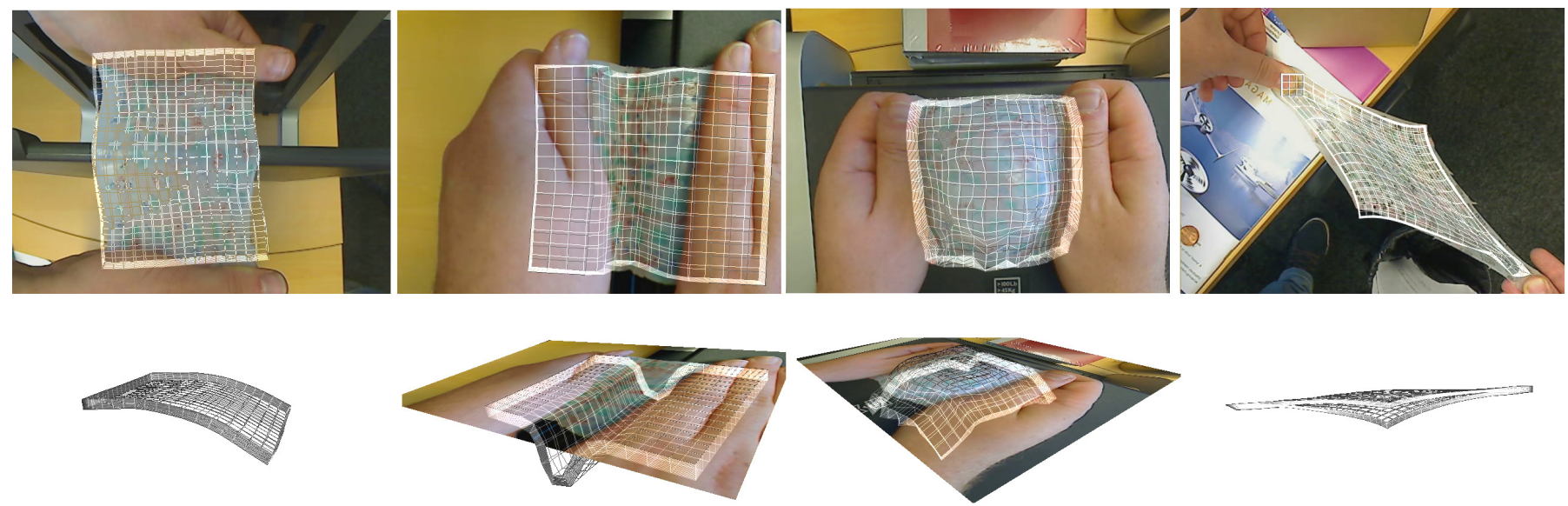

Fig. 1. Three-dimensional reconstruction and augmentation of elastic objects from a single view under several elongations. Our approach is able to handle extensibility of the material when undergoing elongation. (top) camera view of the re-textured elastic object. (bottom) the recovered $3 \mathrm{D}$ shape form showed from a different view.

\section{Mechanical models}

Mechanical models are very popular in both Computer Graphics and Computer Vision. Their ability to translate physical behaviour of objects with fidelity is a major feature for applications such as medical imaging [8], [9] and medical simulation [10]. In computer vision, active contour models [11] have contributed to popularize mechanical-based models. In these models, the internal energy term is an approximation of the elastic deformation and ensures adequate regularization properties of the extracted contours. This method was latter extended to handle volume data [12]. In [13], a dynamic model based on the equation of dynamics for elastic materials is proposed, where forces measured on the image drive the model towards object's boundaries. The organ is supposed to be homogeneous and the forces measured on MRI volumes, though noisy, are available everywhere in the structure.

Most of the time, simple models based on linear elasticity are considered. For tracking heart beats, [5] used a 4D scan of the heart coupled with a biomechanical model. It is controlled by surface constraints created by features extracted from a stereovision stream and allows quite accurate estimation of the deformation [5], [6], [7].

Mechanical models permit an accurate non-rigid registration while producing a coherent visual deformation. Their limitations reside in their complexity and their cost in computing. In addition, a prior knowledge of the object physical properties is often necessary.

\section{Mass-spring Systems}

Mass-spring systems are very intuitive deformable models [15]. In such models, the deformation is approximated by a set of point masses connected by massless springs. Instead of going through a discretization stage, the model offers directly a discrete representation which only requires the solution of a system made of coupled ordinary differential equations.

The main advantage of mass-spring systems is the computational efficiency, since real-time can easily be achieved. Its disadvantage is that it generates non-realistic deformations. Indeed, it is difficult to link the stiffness of the springs to real physical parameters. Furthermore, the resolution of the mesh impacts heavily on the deformations making the model mesh dependent.
Despite these drawbacks, mass-spring systems are used by the research community [16] and in commercial medical simulators.

\section{Learned Non-rigid Models}

Instead of trying to model the physics of the considered object, learning models from a representative set of examples is an interesting alternative which often allows to obtain models with few degrees of freedom. These models have been widely used in computer vision for tracking 2D face deformations using Active Shapes Models [17] or latter Active Appearance Models [18]. They have also been used for 3D non-rigid shape recovery from a single view [19].

Learned non-rigid models are considered very effective for many applications since they do not need prior knowledge of the material parameters. However, gathering enough examples to build a meaningful database requires a huge amount of work and is intractable if highly deformable objects are considered. Automatic generation of plausible shapes in the case of inextensible deformable surfaces has been experimented in [19] for building the database but the method is tightly related to the inextensibility property and cannot be generalized to highly deformable objects.

As an alternative some approaches rely on the use of a regularization parametric shape models. These models use a reduced number of mesh vertices (control points) that interpolate the deformation in order to obtain a finer mesh description.

\section{Parametric Models}

Parametric models are widely used in computer vision as warp functions for image deformations. Their main advantage is to represent the deformation with a small number of control points. A large variety of warp functions exists in the literature, the most popular warps are those based on Radial Basis Functions such as the Thin-Plate Spline (TPS) [20] and those based on the tensorproduct, called Free-Form Deformations (FFD) [21] using cubic B-spline or Bezier's volumes.

Thin-Plate Spline is a radial basis function which minimizes its internal bending energy to model deformations. It has been successfully applied for modeling non-rigid surfaces [22]. TPS is very attractive, thanks to its flexibility in placing control points 
that drive the deformation, thus recent works take advantage of TPS for modeling heart tissue deformation [23], [24].

The basic idea of Free-form Deformation model is to deform an object by manipulating an underlying mesh of control points. Deformations can be imposed with any desired degree of derivative continuity, thus producing smooth transformations. FFD's have been previously applied to tracking and motion analysis in medical imaging [25] and non-rigid surface [26], [27]. A disadvantage of standard FFD is their lack of ability to model local deformations.

Shape Parametrization models are straightforward to implement in real-time and are flexible since they rely on the manipulation of the nodes of the mesh or outlying control points. Nevertheless, these methods do not allow internal properties to be introduced, making them unsuitable for creating realistic biomechanical deformations.

\section{Non-rigid Shape Recovery and Augmenta- TION}

In early works on augmented reality for deformable objects, registration of images of a deforming surface was obtained by computing dense 2D/2D transformations using points correspondences. A parametric representation of the deformation or regularization techniques were needed to prevent excessive wrinkling of the surface in the presence of erroneous correspondences. Bartoli et al. [26] took advantage of a rich texture information to perform points matching between images, allowing a 2D deformation motion model to be computed using Radial Basis Mapping. Pilet et al. [28] proposed a template-based fast and robust tracking for handling deformations. This approach uses a set of wide baseline feature matches assuming a well textured surface and combines 2D deformable meshes with a robust estimation technique. Zhu et al. [29] demonstrated that a Finite Newton algorithm and an efficient factorization method can reduce the number of iterations of the previous method to solve the optimization problem. In order to handle the more challenging case of deformation with self occlusions, Gay-Belille et al. [30] considered the occluded pixels as a self-occlusion area that forces the wrap to shrink instead of outliers. Inspired by this self-occlusion shrinking method, Hilsmann et al. [31] proposed an approach exploiting an optical flow extended by a specific illumination model which jointly estimates deformation and illumination and can cope with self-occlusions through an occlusion map computed from local statistical color models. In [27], self occlusions are detected as outliers based on the assumption that the surface to detect is locally smooth.

However, these methods based on 2D image transformations are well suited to smooth deformations but are not suitable for highly elastic objects. In fact, elastic deformations in the 3D space can lead to highly complex 2D deformations in the image plane, especially due to self occlusions, making inappropriate the use of regularization constraints. For these reasons, state-of-the-art methods perform 3D reconstruction of deformable surfaces in a monocular context since elasticity constraints are often known or can be expressed in a more natural way on the $3 \mathrm{D}$ objects. Recovering the 3D shape of a deformable surface from a monocular video and a template (a reference image of the surface for which the 3D shape is known) can be ambiguous. Therefore, additional consistency constraints are required to solve ambiguities. The inextensibility constraint is widely used to recover and augment objects as paper sheets, sails, tee-shirts [32], [33]. Methods differ in the way inextensibility is considered as a hard constraint or a penalty term and also in the efficiency of the convex or nonconvex optimization associated to the procedure. In the general case, global smoothness constraints are common to resolve the ambiguities of 3D reconstruction. Many papers resort to a linear description of feasible object deformations. Most of the time, it is generated from a representative sample of possible shapes using a dimensionality reduction process [19], [34].

Other methods have been designed to cope with non smooth deformations, as folding. Salzmann et al. [35] proposed to solve the problem as a convex minimization of the reprojection error formulated as a Second Order Cone Programming. The method restricts the motion from one frame to the next but do not impose unwarranted surface smoothness, making it possible to recover sharp folds. With a similar method the convex minimization can also be formulated as a Quadratic Programming [32] in order to reduce the sensitivity to outliers.

Some works attempted to overcome the need to provide plausible constraints on the deformation by using richer sources of information. In [36] a closed-form solution constrained by shading information was introduced to capture stretching surface. This method assumes a Lambertian surface with a single point light source and yields good results. However, the strong assumptions on the lightening make the method hard to generalize in all environments.

Recently, many methods using mechanical-based tracking have emerged. For instance the approach in [37], where a combination of Finite Element Modelling with an Extended Kalman Filter shows the efficiency of physics-based methods. In [38], a linear finite element method is used to predict the deformation. The approach described in [39] relies on the minimization of a stretching energy subject to external image constraints. The problem is formalized as a non-linear minimization that unifies geometric constraints assuming a projective camera and mechanical constraints assuming local linear elasticity. This method shows effective results considering the Poisson ratio as the unique parameter.

\section{CONTRIBUTIONS}

This paper is an extension of our previous work published in [40] where we proposed an efficient method for the 3D reconstruction and augmentation of elastic surfaces, using only single view images (see Figure 1). The main contributions of this paper are:

- $\quad$ an efficient method to capture and augment a 3D elastic surface from a single viewpoint. By assuming a prior knowledge of the material elasticity, large strains of more than $130 \%$ can be efficiently handled. To the best of our knowledge, no similar method has been proposed in the case of monocular camera

- handling significant self-occlusions by introducing a generic constraint based on the equal distribution of the image stretching forces. Our method is qualitatively and quantitatively assessed with self-occluded surfaces with up to $60 \%$ of visible mesh.

- a comparison to existing techniques with experiments conducted on computer-generated and real data that show the effectiveness of our approach. Experiments in the context of minimally invasive liver surgery are also provided. We conduct experiments on the importance of boundary conditions in the establishment of a well-constrained problem. 


\section{Non Linear Elastic model}

The choice of a relevant constitutive model is essential as it will determine the set of deformations we are able to capture and estimate while discriminating non-plausible material configurations induced by tracking errors. Two important assumptions are also made in order to reduce the complexity of the deformation model and the number of related parameters. First, the material of the deformable object will be homogeneous meaning that a uniform deformation will lead to equal (in magnitude) forces for any point of the object. Second, the material will also be considered as isotropic meaning that the response to a deformation is independent of the orientation of the deformation. Moreover, the computation time is also a key constraint as the targeted application should be interactive. Interactive (or at least computationally fast) models for deformable solids have been a major topic in the computer graphics community. Several reports or surveys provide an exhaustive overview of state-of-the-art methods [41], [42], [43]. In this context, the Saint Venant-Kirchhoff model [43] appears to be an ideal compromise because it is able to handle non-linear deformations, is rotationally invariant and is simple enough compared to other non-linear models and therefore can be computed at interactive rates. Several downsides however exist such as incorrect stress estimation under extreme compression and sometimes the requirement to use non-linear solvers to compute the motion over time. Incorrect stress estimation under large deformations is not a major issue in our context since we are interested in capturing an accurate deformation field and since the stress field is not measurable with camera images. Recent works in simulation or in haptics rendering have proposed computationally fast nonlinear solvers such as [1] which leverage the pre-requisites of using non-linear solvers with Saint Venant-Kirchhoff model.

A Saint Venant-Kirchhoff (StVK) material is a material for which the Green-Lagrange strain tensor $\mathbf{E} \in \mathbb{R}^{3 x 3}$ is computed as a non-linear (quadratic) function of the deformation gradient $\mathbf{F} \in \mathbb{R}^{3 \times 3}$ as:

$$
\mathbf{E}=\frac{1}{2}\left(\mathbf{F}^{T} \mathbf{F}-\mathbf{I}\right)
$$

where $\mathbf{I} \in \mathbb{R}^{3 \times 3}$ is the identity matrix. The computation of the strain tensor may be computationally intensive and several approaches have been investigated for interactive uses. For instance, Barbic and James [44] use model reduction and precomputation reduced coordinates while Zhong et. al. [45] uses pre-computed relations between surface and internal nodes. In this paper, the elegant approach of Kikuuwe et. al. [46] is chosen since it does not require pre-computation nor does it make any assumption of the deformations that will be generated. To do this, fast computation is conducted using dedicated yet simple data-structures (tetrahedron-sharing edge-pairs). We will detail the main points of Kikuuwe $e t$. al. but we encourage the reader to refer to [46] for complete analysis and implementation details. If $v$ is a point in the deforming medium and $v_{0}$ is its initial location, $\mathbf{F}$ can be expressed as $\mathbf{F}=\frac{\partial v}{\partial v_{0}}$. Since $\mathbf{E}$ is symmetric, its form is as follows:

$$
\mathbf{E}=\left[\begin{array}{lll}
\varepsilon_{x x} & \varepsilon_{x y} & \varepsilon_{z x} \\
\varepsilon_{x y} & \varepsilon_{y y} & \varepsilon_{y z} \\
\varepsilon_{z x} & \varepsilon_{y z} & \varepsilon_{z z}
\end{array}\right]
$$

and the 6 independent coefficients may be placed in a single vector $\varepsilon$ using Voigt notation:

$$
\varepsilon=\left[\varepsilon_{x x}, \varepsilon_{y y}, \varepsilon_{z z}, 2 \varepsilon_{x z}, 2 \varepsilon_{y z}, 2 \varepsilon_{z x}\right]^{T}
$$

Computing $\mathbf{E}$ is often conducted by using a strain energy density $w$ that will be integrated over the whole deforming medium $X$ as follows:

$$
\mathbf{E}=\int_{X} w d X
$$

An isotropic homogeneous Saint Venant-Kirchhoff material has $w$ expressed with the following equation:

$$
w=\varepsilon^{T} \mathbf{D} \varepsilon / 2
$$

where $\mathbf{D} \in \mathbb{R}^{6 x 6}$ is the following matrix:

$$
\mathbf{D}=\left[\begin{array}{cccccc}
\lambda+2 \mu & \lambda & \lambda & 0 & 0 & 0 \\
\lambda & \lambda+2 \mu & \lambda & 0 & 0 & 0 \\
\lambda & \lambda & \lambda+2 \mu & 0 & 0 & 0 \\
0 & 0 & 0 & \mu & 0 & 0 \\
0 & 0 & 0 & 0 & \mu & 0 \\
0 & 0 & 0 & 0 & 0 & \mu
\end{array}\right]
$$

where $\lambda$ and $\mu$ are Lamé coefficients and can be computed thanks to the elastic parameters of the material $E$ and $v . E$ is the Young's modulus and is a measure of the stiffness of the material while $v$ is the Poisson's ratio and estimates the compressibility of the material.

For a deforming medium of an arbitrary shape, it is often convenient to discretize the shape using elementary and simple elements. Tetrahedral decomposition is often considered since efficient meshing algorithms exist (for instance CGAL library [47]). For a tetrahedron $t$, let us consider the edge $e$ among the 6 possible edges of $t, e$ being connected to two vertices $v_{i}$ and $v_{j}$. Be $l_{e}=v_{i}-v_{j}$ and $l_{e}^{0}=v_{i}^{0}-v_{j}^{0}$. Assuming that the deformation gradient $\mathbf{F}$ is constant in the neighborhood of $t$ and has the value $\mathbf{F}_{t}, l_{e}$ can be estimated as $\mathbf{F}_{t} l_{e}^{0}$. Equation (1) can be used to write:

$$
\begin{aligned}
l_{e}^{T} \mathbf{E}_{t} l_{e} & =\frac{1}{2} l_{e}^{T}\left(\mathbf{F}_{t}^{T} \mathbf{F}_{t}-I\right) l_{e} \\
l_{e}^{T} \mathbf{E}_{t} l_{e} & =\frac{1}{2}\left(\left\|l_{e}\right\|^{2}-\left\|l_{e}^{0}\right\|^{2}\right)
\end{aligned}
$$

$l_{e}^{0}$ can also be written in Voigt notation using the variable $q_{e}^{0}$ defined as:

$$
q_{e}^{0}=\left[\begin{array}{c}
\left(v_{i} \cdot x-v_{j} \cdot x\right)^{2} \\
\left(v_{i} \cdot y-v_{j} \cdot y\right)^{2} \\
\left(v_{i} \cdot z-v_{j} \cdot z\right)^{2} \\
\left(v_{i} \cdot x-v_{j} \cdot x\right)\left(v_{i} \cdot y-v_{j} \cdot y\right) \\
\left(v_{i} \cdot y-v_{j} \cdot y\right)\left(v_{i} \cdot z-v_{j} \cdot z\right) \\
\left(v_{i} \cdot z-v_{j} \cdot z\right)\left(v_{i} \cdot x-p_{j} \cdot x\right)
\end{array}\right]
$$

where $. x, . y, . z$ are the first, second and third component of the considered vector. The previous equation is then rewritten using Voigt notations as:

$$
q_{e}^{0 T} \varepsilon_{t}=\frac{1}{2}\left(\left\|l_{e}\right\|^{2}-\left\|l_{e}^{0}\right\|^{2}\right)
$$

This equation is considered for a single edge $e$ but is suitable for the other edges. By considering the 6 edges of the tetrahedron $t$, we obtain the following equation: 


$$
\mathbb{Q}_{t} \varepsilon_{t}=\frac{1}{2} \mathbb{L}_{t}
$$

where $\mathbb{Q}_{t}=\left[q_{0}^{0}, q_{1}^{0}, q_{2}^{0}, q_{3}^{0}, q_{4}^{0}, q_{5}^{0}\right]^{T} \quad\left(q_{e}\right.$ for the 6 possibles edges) and $\mathbb{L}_{t}=\left[\left\|l_{0}\right\|^{2}-\left\|l_{0}^{0}\right\|^{2},\left\|l_{1}\right\|^{2}-\left\|l_{1}^{0}\right\|^{2}, \ldots\right]^{T}$ (again for the 6 possible edges). It should be noted that $\mathbb{Q}_{t} \in \mathbb{R}^{6 x 6}$ and $\mathbb{L}_{t} \in \mathbb{R}^{6}$. $\mathbb{Q}_{t}$ is invertible if the 4 points are not linearly dependent and therefore $\varepsilon_{t}$ can be estimated as:

$$
\varepsilon_{t}=\frac{1}{2} \mathbb{Q}_{t}^{-1} \mathbb{L}_{t}
$$

enabling to compute the strain energy in the tetrahedron $t$ with

$$
W_{t}=\varepsilon_{t}^{T} \mathbf{D} \varepsilon_{t} / 2
$$

Therefore the total strain energy $W=\sum W_{t}$ of the medium is expressed as a function of the edge lengths of the tetrahedral mesh and elastic parameters. Eventually the forces exerted on the vertices can be computed as:

$$
f(v)=\frac{\partial W}{\partial v^{T}}
$$

For computation purposes, a global stiffness matrix is also computed as:

$$
K(v)=\frac{\partial f(v)}{\partial v}
$$

as it allows for a displacement $d v$ (such as $\left.v^{\prime}=v+d v\right)$ of the vertices of the mesh to compute the resulting forces as:

$$
f(v)=K(v) \times d v
$$

This is a convenient equation that relates forces to any displacement of the vertices but it should be reminded that the matrix $K(v)$ should be recomputed after every deformation to remain valid.

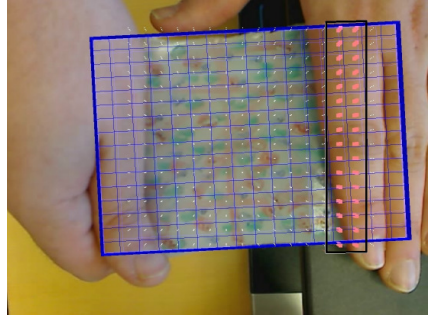

(a) Deformation 1

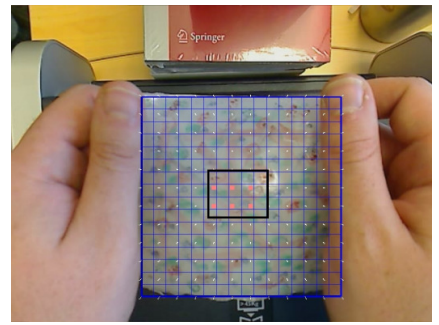

(c) Deformation 3

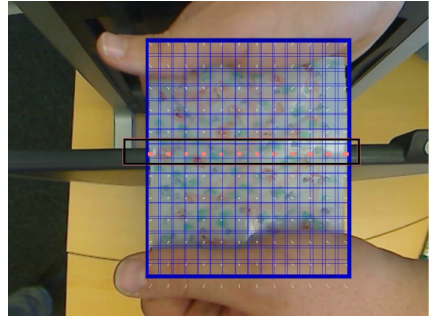

(b) Deformation 2

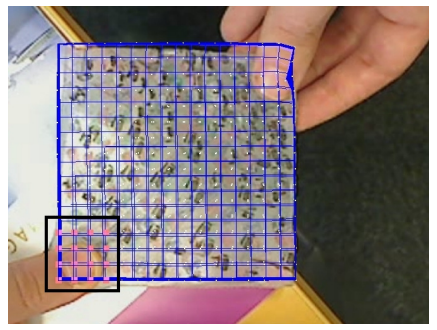

(d) Deformation 4
Fig. 2. Silicone Dataset. The mesh is represented in blue and the boundary conditions in red surrounded by a black square.

\section{Problem Formulation}

Our work takes place in the context of template based reconstruction from monocular images. Given a reference image for which the $3 \mathrm{D}$ shape is known, we want to infer the $3 \mathrm{D}$ shape deformation from image correspondences detected in the video flow. Assuming a known and constant projection matrix $P$, the projection of a point $m^{*}=\left(x^{*}, y^{*}, z^{*}\right)$ in the image is the point $m=(x, y)$ given by:

$$
m=P m^{*}=\left(\begin{array}{c}
\frac{P_{1} m^{*}}{P_{3} m^{*}} \\
P_{2} m^{*} \\
P_{3} m^{*}
\end{array}\right)
$$

where $P_{k}$ is the $k^{\text {th }}$ row of the projection matrix $P$.

As shown in [34], formulating the $3 \mathrm{D}$ recovery of a triangulated surface as the minimization of the reprojection error of the tracked features is an underconstrained problem. Additional knowledge must thus be introduced to recover properly the deformation.

In practice, additional penalty functions are used to obtain a well-constrained system. These constraints are often based on the inextensibility property of the surface to be recovered. Since such constraints cannot be considered in our case, we propose to consider the elastic registration as a stretching energy minimization problem that accounts for the internal forces of the mechanical and external forces emanated from the visual tracking.

\section{Required assumptions}

In order to ensure a good shape recovery and a well-posed system, we assume that the following data are available:

- The projective matrix $P$ assuming a fixed camera.

- The correct initial alignment of the mesh (in rest configuration) on the image.

- $\quad$ A set $T$ of paired points $U^{*}=\left\{u_{i}^{*} \in \mathbb{R}^{3}\right\}_{\{i \in T\}}$ between the set of features $U=\left\{u_{i} \in \mathbb{R}^{2}\right\}_{\{i \in F\}}$ from visual tracking and the mesh vertices $V=\left\{v_{i} \in \mathbb{R}^{3}\right\}$ related to the mechanical model.

- The material stiffness that represents the elasticity of the mechanical model.

\section{Initialization}

At initialization, each feature $u_{i}$ is associated with a $3 \mathrm{D}$ point $u_{i}^{*}$ by intersecting the line of sight with the surface. $u_{i}^{*}$ is expressed as a barycentric combination of facet vertices as follows:

$$
u_{i}^{*}=a_{i} v_{1}+b_{i} v_{2}+c_{i} v_{3}
$$

where $v_{i ; 1 \leq i \leq 3}$ are the vectors of $3 \mathrm{D}$ vertex coordinates and $\left(a_{i}, b_{i}, c_{i}\right)$ the barycentric coordinates of $u_{i}^{*}$. We assume that this linear relation remains valid during the deformation.

\section{Image Fitting}

We propose to consider the features displacement as a stretching energy defined as

$$
E_{S}=\sum_{i \in F} \frac{1}{2} k\left\|u_{i}-P\left(u_{i}^{*}\right)\right\|^{2}
$$

where $k$ can be seen as a stiffness and is chosen to be the same order of magnitude of the Young's modulus. 


\section{Boundary Conditions}

In order to obtain a system that is sufficiently constrained to give good results, we add to the system a set $B$ of boundary constraints using the hard constraint:

$$
v_{i}=Q_{i} \text { for } i \in B
$$

where $Q_{i}$ are the known boundary conditions that can be seen as a set of fixed vertices. The boundary conditions are necessary to yield good results. These boundary conditions are most of the time quite natural and specify how the object is linked to its environment such as an object fixed to a table or contained by an obstacle (illustrated in the Figure 2).

\section{Energy Minimization}

The minimization problem is then formulated as a constrained minimization between the internal elastic energy and stretching energy and can be written as follows:

$$
\begin{aligned}
\min E(v) & =\sum_{t} W_{t}+\sum_{i \in F} \frac{1}{2} k\left\|u_{i}-P\left(u_{i}^{*}\right)\right\|^{2} \\
\text { subject to } & v_{i}=Q_{i} \text { for } i \in B
\end{aligned}
$$

where $W_{t}$ is the strain energy of a tetrahedron related to a Saint Venant-Kirchhoff material and which depends on the position of the vertices. The expression of $W_{t}$ is detailed in equation (12).

\section{Resolution}

Equation (20) is a classical constrained minimization problem. We choose to solve this equation by formulating a linear complementary problem (LCP) that will be solved with a Gauss-Seidel algorithm using the approach in [48]. Finding the minimum of energy is conducted by deriving and setting to zero the equation (20). This leads to an equality between internal elastic forces and stretching forces and this is equivalent finding the equilibrium state of the material due to external forces. Indeed the kinetic energy is not considered since we are not interested in capturing transient motions because first, the materials used are very soft and second, the acquisition rate is high enough; therefore the deformation exhibits no significant transient motion. Even if our approach is static (without dynamic due to kinetic energy of the material), it can easily be adapted to dynamic motion with the same method by adding a non-linear differential equation solver such as an implicit Euler with a conjugate gradient. In other words, this amounts to solve the linear system $\mathbf{A} \cdot \mathbf{x}=\mathbf{b}$ given by integrating in time the equation of dynamic $\mathbf{M a}=\mathbf{f}$, where $\mathbf{f}$ includes the external forces $f_{s}$ derived form the stretching energy $E_{s}$. Since the model used here is homogeneous, one can rely on a conjugate gradient iterative solver where pre-conditioning is possible to solve the linear system [10].

\section{Dealing with Self-occluded Regions}

Solving the system of equation (20) yields to recover the 3D shape that will satisfy both physical and geometrical constraints, and thus by finding the equilibrium between internal and external forces. However, when dealing with occluded regions, extra care has to be taken in the expression of external loads. Indeed, the physical model can be over-constrained by erroneous external forces and can be forced to fit occluded features that are subject to erroneous positions. This can highly damage the recovered shape as illustrated in Figure 3.

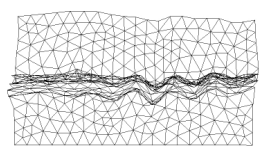

(a) Top view

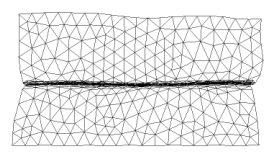

(d) Top view

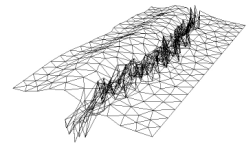

(b) Perspective view

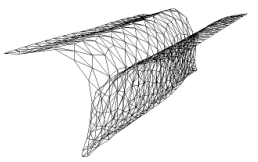

(e) Perspective view

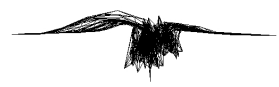

(c) Front view

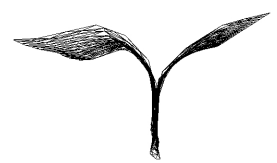

(f) Front view
Fig. 3. The inputs to the self-occlusion problem are the unreliable feature points that damage the mesh with (a), (b) and (c) the recovered mesh under different view without handling self-occlusions and (d), (e) and (f) the mesh under different views with our generic constraint based on equal force distribution to handle self-occlusions.

In order to resolve occlusions, several strategies have been considered. Some rely on a preliminary stage that aims to reject outliers directly from the input image, this leads to detect the occluded map on the mesh [27]. Other methods detect selfocclusions as warp shrinkage areas where the warp is constrained to shrink rather than to fold [30], whereas visual consistency can also be considered in multiframe reconstruction [49].

These approaches assume strong prior on the type of deformation, which can hardly be considered for elastic objects where the number of degrees of freedom is high, or relies on a twostage processing to detect occluded areas. We introduce here a weak, computationally cheap and generic constraint that easily fits into our framework. By taking advantage of the capability of the physical model to estimate 3D shape where no external loads are present, we reduce the number of features according to the resolution of the underlying mesh, instead of simply evicting outliers. Whereas in previous approaches the number of features might not be large enough to recover self-occluded regions, it permits in our case to equally distribute the stretching forces to ensure a global physical consistency. We thus select among the set of mapped features $U^{*}$ a subset of features with high detection scores at the corresponding $u_{i}$ and impose a minimal relative distance between them:

$$
\left\|u_{i}^{*}-u_{j}^{*}\right\| \geq \tau
$$

where $u_{i}^{*}$ is the closest neighbour of $u_{j}^{*}$ and $\tau$ is the initial length of the edge corresponding to the largest tetrahedral element of the model. Locally selecting the features according to their detection scores make the tracking and the detection of possible tracking failures easier, thus limiting the introduction of outliers in the visual features. Contrary to existing approaches which require many features for correctly handling occlusions, we take advantage of the ability of the mechanical model to predict the shape in the areas with unobserved data, especially in occluded areas, and select a set of reliable and roughly equally distributed features to guide the mechanical model. This constraint has only to be considered at initialization, where the mesh is at its rest configuration, thereby, the extensibility property of the material remains valid and self-occlusions are efficiently handled, as will be shown in Section 9. 


\section{Results}

In this section we present the results obtained using our method and the comparison conducted with existing techniques. We report results obtained on three types of data: Silicone-made data with and without self-occluded areas, computer-generated data and liver data during in-vivo surgical procedure. To test the ability of our approach to capture 3D elastic deformations, we capture several video sequences of a silicone-like object undergoing different types of stretching deformation. We quantify the threedimensional shape recovery error with respect to a ground truth while visual assessment is reported on surgical data. We use SURF [50] for establishing image point correspondences where the number of detected points can be easily tuned. Note that other descriptors can easily be plugged to the framework and take advantage of recent work on vision-based tracking of living tissues [51]. Note also that the implementation is done in $\mathrm{C}++$ and runs on an off-the-shelf PC with and Intel i7 M620 2.76GHz processor.

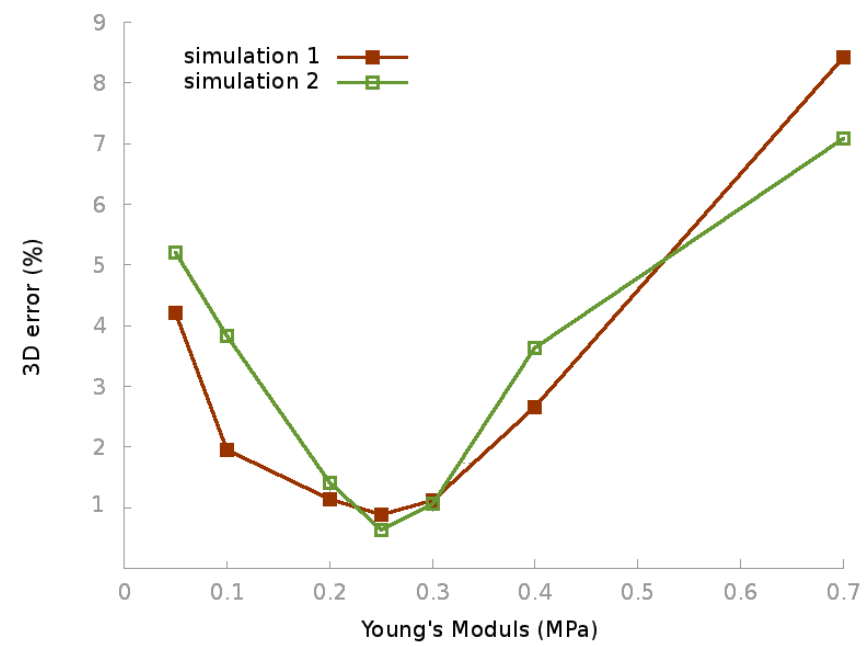

Fig. 4. 3D registration error with variation of the Young's Modulus for simulation 1 and simulation 2: Small variations of the Young's Modulus value slightly affects the reconstruction while distant values highly increase the error.

\subsection{Computer-generated Data}

We used the framework Sofa [52] to generate elastic deformations of a silicone-like object with a Young's Modulus of $E=0.25 \mathrm{MPa}$. A force $F$ is applied on the object to produce a 3D deformation (cf Figure 5). A video sequence of the deformations is captured with a virtual camera $(\operatorname{diag}(500,500,1))$. The external stiffness parameter is set to be equal to the modulus of the synthetic force. Figure 5 illustrates the results obtained by calculating the Hausdorff Distance between the tracked and the simulated mesh where we report an average overlay error of $0.83 \%$ for simulation 1 and $0.70 \%$ for simulation 2 .

\subsubsection{Parameters Sensitivity}

We also conducted experiments on the same set of data where we varied the Young's Modulus value. The plot in Figure 4 shows that small variations of $E$ slightly affects the registration error in comparison to the large errors that produce greater or lower values. In the mean time, we notice that a value of $k$ close to the Young's Modulus gives a better accuracy.

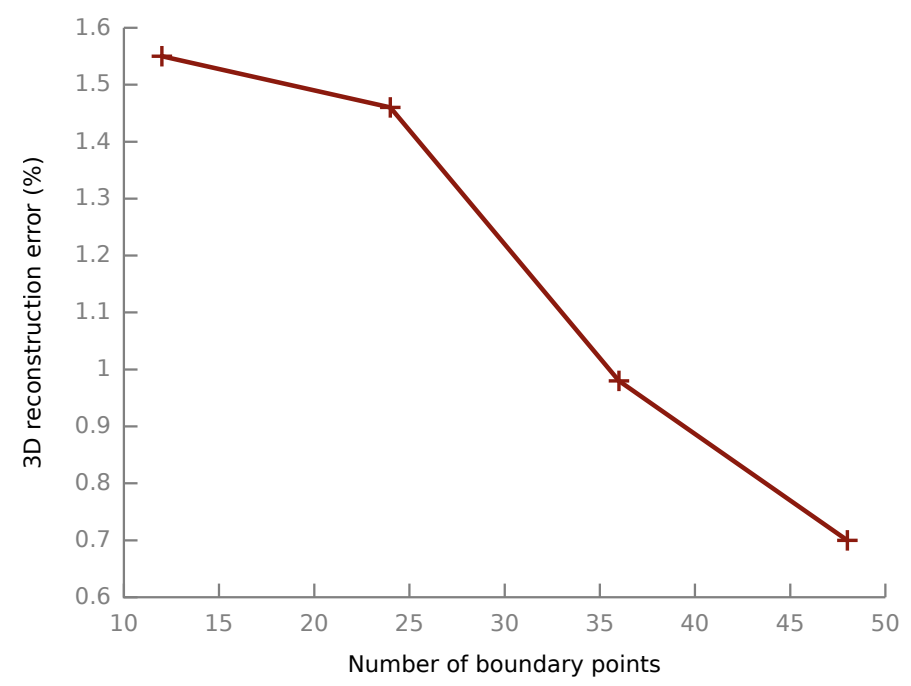

Fig. 6. Impact of the boundary conditions on the 3D reconstruction.

It should be emphasized that a further sensitivity study is required to identify the most influential parameters of the simulation [53]. The conducted experiments only highlight that an approximate value of the Young's Modulus is enough to yield convincing registration results. In fact, depending on the way the constraints on the deformable structure are modeled, the resulting deformation can be entirely independent of the Young's Modulus. The reader may refer to [54] for a more detailed study on the impact of the material properties when dealing with soft-tissue simulation.

\subsubsection{Boundary Conditions}

We take advantage of synthetic data to assess the necessary use of boundary conditions (see eq 19) to reach an acceptable shape recovery. We plot in Figure 6 the 3D reconstruction error when varying the number of boundary points from 12 to 48 points. The results show that the boundary conditions are necessary to yield a good shape recovery and resolve ambiguities.

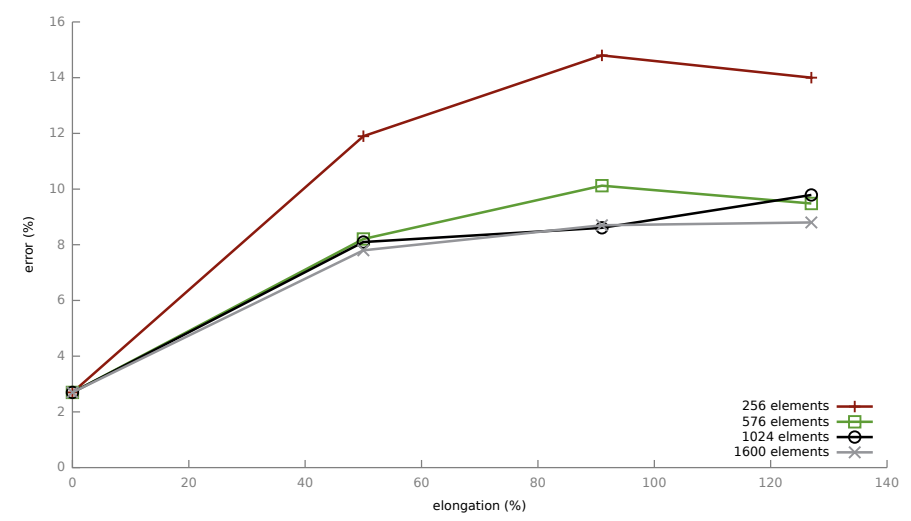

Fig. 7. Registration error with respect to object elongation with a variation of mesh resolution: using an adequate number of tetrahedral elements the projection error can be significantly reduced.

\subsection{Silicone Data}

We tested our method on a silicone-like object with a size of $100 \times 100 \times 10 \mathrm{~mm}^{3}$ composed of linear P1 tetrahedral elements 


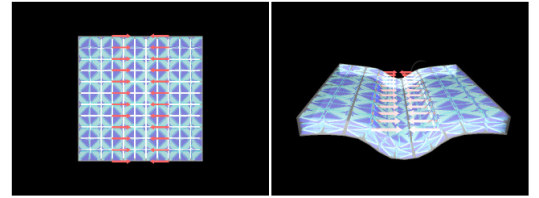

(a)

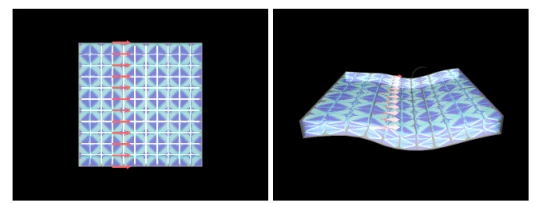

(e)

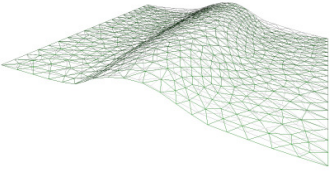

(b)

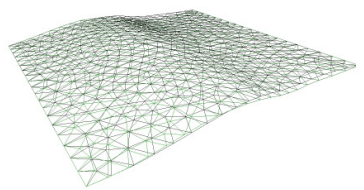

(f)

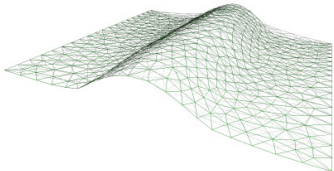

(c)

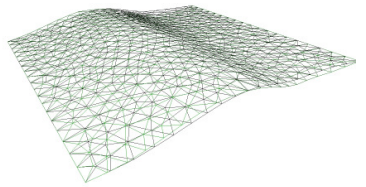

(g)

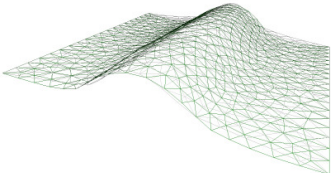

(d)

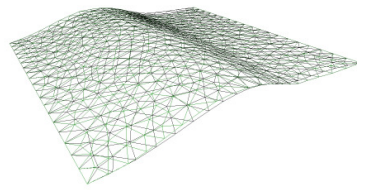

(h)

Fig. 5. 3D registration error on two computer-generated sequences. The applied forces are represented with red arrows and the tetrahedral elements in blue, for (a) simulation 1 where two forces in opposite directions are applied and (b) simulation 2 where a single force is applied.

characterized by a Young's Modulus $E=0.25 \mathrm{MPa}$. The images were acquired with a monocular camera at $30 \mathrm{fps}$ with an image resolution of $640 \times 480$.

The ground truth is obtained using Structure from Motion techniques [55]. At the end of each manipulation, we reconstruct the $3 \mathrm{D}$ scene with fiducials placed around the silicone object. These fiducials are necessary to align the 3D mesh on the input image (last frame of the sequence) in order to scale the mesh and apply the necessary rotation and translation. Some errors may occur from these transformations, in addition to errors that emanate from the occluded regions, but it is worth mentioning that the same ground truth is used for the comparison.

\subsubsection{D Surface Registration and Retexturing}

The aim of conducting tests on a $2 \mathrm{D}$ surface is to measure the registration error w.r.t the deformation of the object. Since the boundaries of the object can be easily extracted from the image, a way to quantify this error is to calculate the amount of overlay (in percentage) between the silhouette computed from the recovered $3 \mathrm{D}$ object and the actual boundaries extracted from the image (see Figure 8). The results reported in Figure 7 give small 2D errors even when the elongation increases (more than 120\%). The relation between the accuracy of the augmentation and the number of tetrahedron elements is also reported in Figure 7 where we can notice that a finer mesh resolution reduces the registration error.
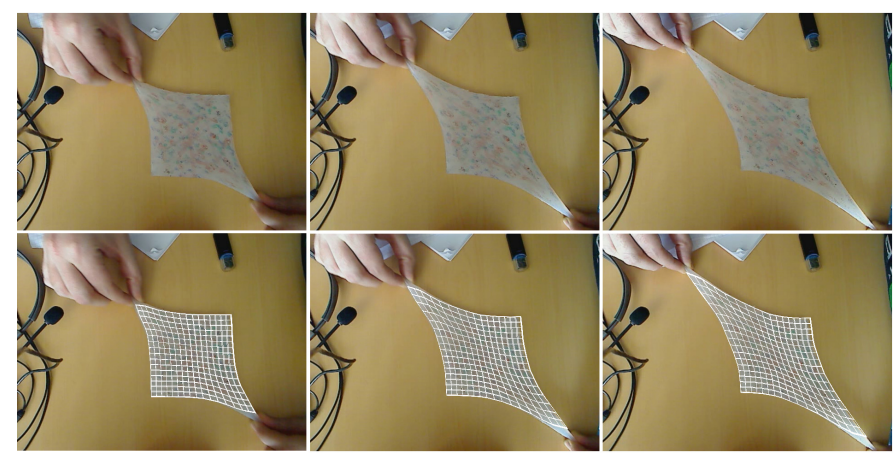

Fig. 8. Selected frames during a 2D elastic surface augmentation of the silicone-like material when being stretched up to $120 \%$, with (top) input images and (bottom) registered mesh.

In order to ensure real-time achievement, a compromise has to be found between the number of elements and the computation time. While a large number of elements permits to obtain an accurate registration, it also increases the computation time. Table 1 gives the average errors and computation time w.r.t the mesh resolution.

\begin{tabular}{lcccc}
\hline Number of elements & 256 & $\mathbf{5 7 6}$ & 1024 & 1600 \\
\hline Average 2D error (\%) & 10.84 & $\mathbf{7 . 6 2}$ & 7.3 & 6.92 \\
\hline Frame rate (fps) & 29 & $\mathbf{1 7}$ & 9 & 5 \\
\hline & TABLE 1 & & &
\end{tabular}

Impact of the number of elements on the computation time.

\subsubsection{D Shape Recovery and Augmentation}

For the three-dimensional reconstruction we propose to test our approach on 4 types of deformation, with extensibility up to $130 \%$ as illustrated in Figure 2. We calculate the Euclidean distance between the reconstructed surface and a ground truth obtained with Structure from Motion techniques [55]. We compare our method with 3 existing approaches: a template-based method for isometric and conformal surface reconstruction described in [56] the code of which is freely available, a classic mass-spring model and a mechanical-based approach that consider a linear Strain/Stress formulation [57]. The plots and the resulting shapes are illustrated in Figures 9, 10, 11 and 12.

Deformation 1: By stretching the object with an elongation of $30 \%$, we force the surface to fold. The linear FEM and the template-based approach fail to correctly recover the 3D shape. The non-linear method gives the lowest error with $2.62 \mathrm{~mm}$ with only 26 detected features.

Deformation 2: The surface is constrained with a rigid beam and stretched to produce a triangle-like shape. Only the mass-spring model was not able to recover the deformation. The template-based approach yields good results. However, the mechanical methods report the most accurate registration with very close error: $2.52 \mathrm{~mm}$ for the Linear FEM and $2.44 \mathrm{~mm}$ for our method. The number of features extracted is 28 for all the methods except the template-based method where 237 features were necessary to yield good results.

Deformation 3: The object is elongated down and constrained at the center. The four methods succeed to recover the shape deformation with 174 extracted features. Our method outperforms the others with an error of $2.06 \mathrm{~mm}$. 


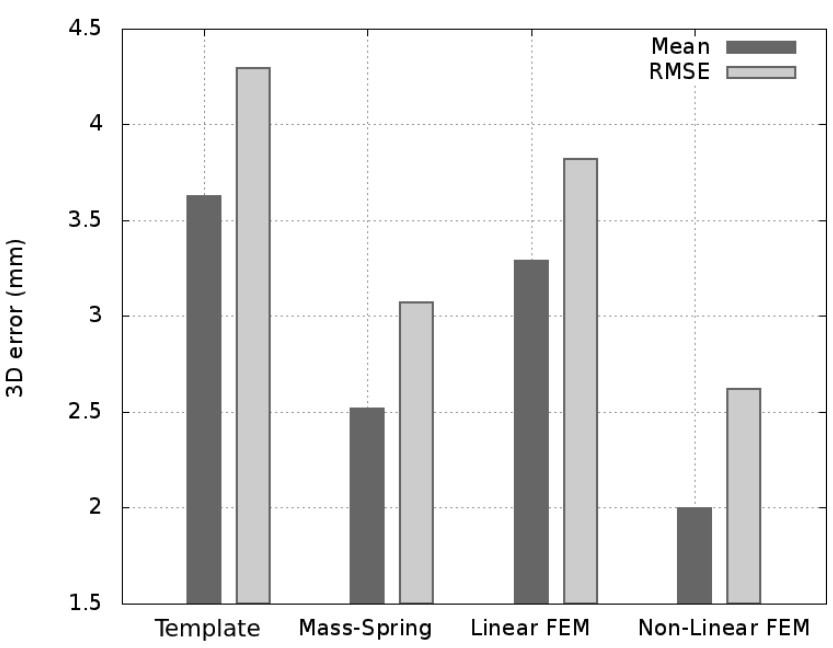

(a)

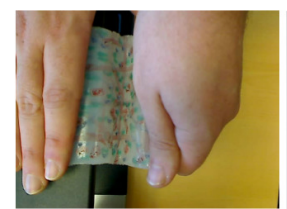

Last Frame

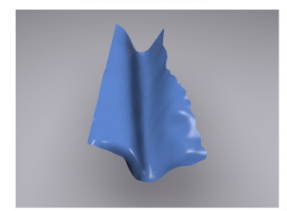

Ground Truth

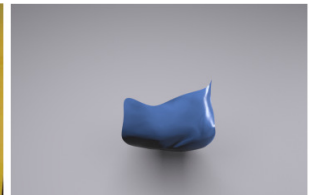

Template-based

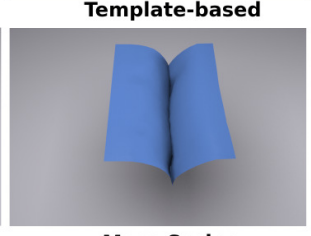

Mass-Spring

(b)
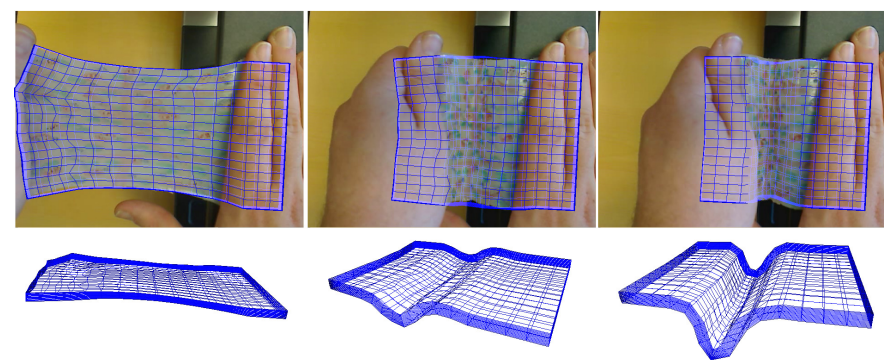

(c)

Fig. 9. 3D shape recovery of a silicone-like material deformation: Our method produces the lowest error in comparison with other methods on deformation 1.

Deformation 4: Our approach gives the smallest error of $1.69 \mathrm{~mm}$ where the extensibility is about $130 \%$. The object highly elongated during the deformation and produced a 3D shape by partially folding. The template-based method fails to recover the stretched shape while the mass-spring and linear FEM models give relatively good shape representations.

\section{Minimally Invasive Surgery}

Our aim is to assess the robustness of our approach in a real environment (specular lights, beating heart, respiratory motion, instrument occlusions) and the ability of our non-rigid registration to recover 3D shapes from a single view. In our past works on liver augmentation [4], we mainly considered deformations of

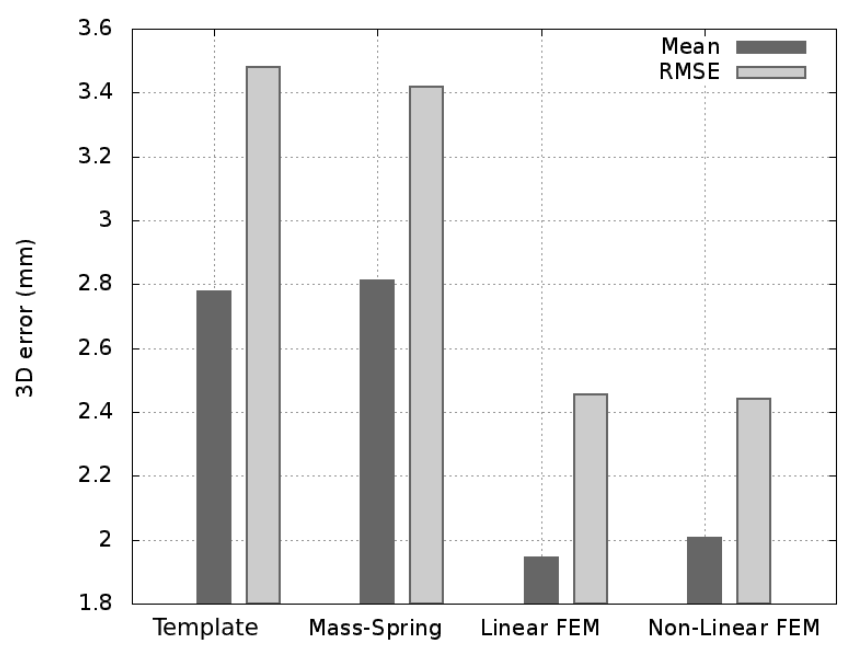

(a)

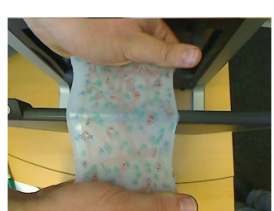

Last Frame

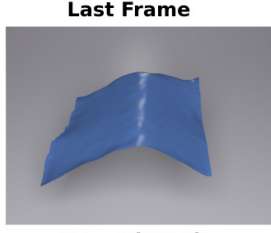

Ground Truth
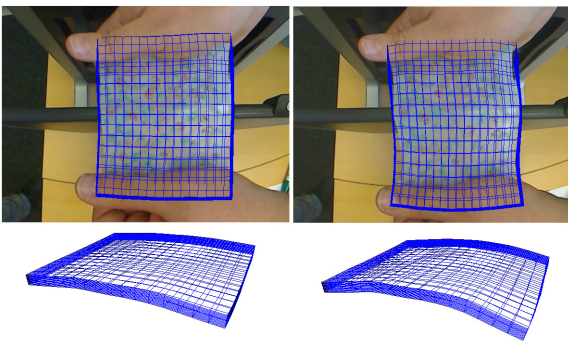

(c)

Fig. 10. 3D shape recovery of a silicone-like material deformation: Our method produces the lowest error in comparison with other methods on deformation 2.

the liver that occur in a fronto-parallel plane. In addition, visual features were acquired in 3D thanks to a stereovision set-up. Here, a general motion of the liver is considered, with possible folds, in the monocular context which is a much more complex situation.

\subsection{Liver Soft-tissue Tracking}

We have tested our approach on a video of in-vivo porcine liver showing one of the liver lobes undergoing an elastic deformation during a minimally invasive surgery. We used a monocular laparoscopic camera from Karl Storz Endoscopy acquiring a video stream of 25 FPS with an image resolution of $720 \times 576$ pixels. The camera is calibrated in an offline stage.

To estimate the motion of the surface of the liver, salient landmarks are detected and tracked over time. Many methods 


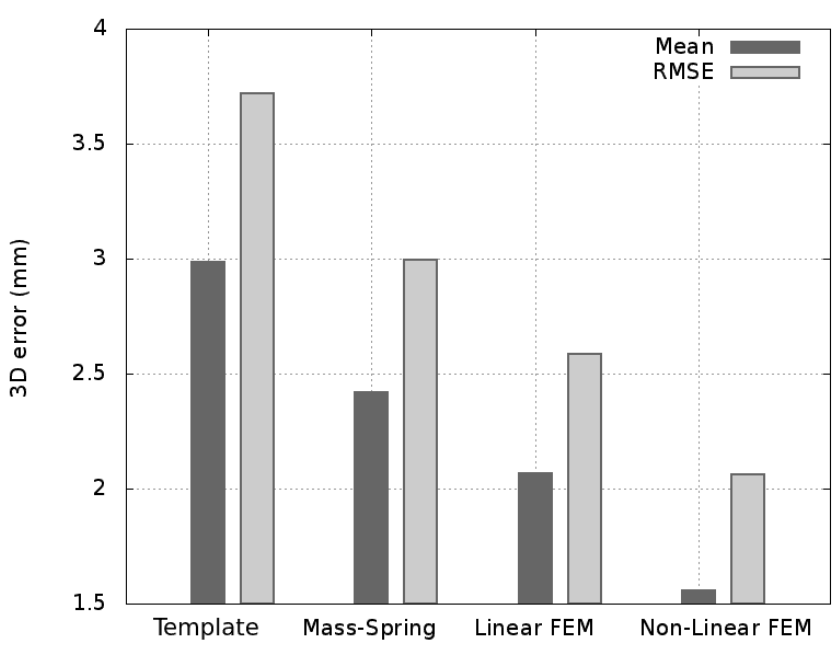

(a)

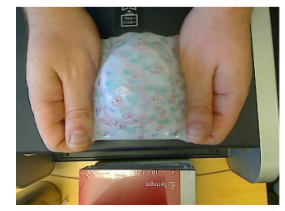

Last Frame

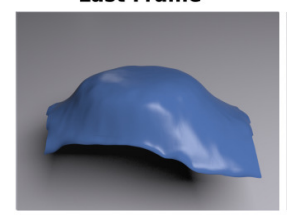

Ground Truth

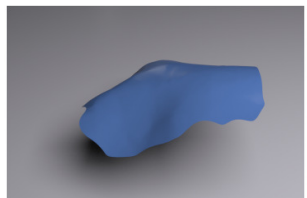

Template-based

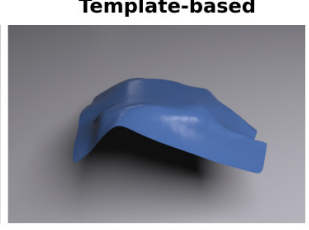

Mass-Spring

(b)
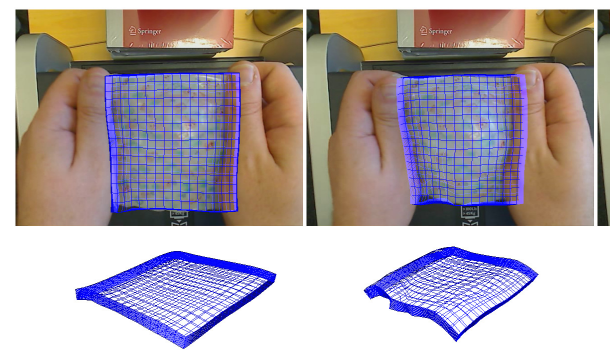

(c)

Fig. 11. 3D shape recovery of a silicone-like material deformation: Our method produces the lowest error in comparison with other methods on deformation 3.

have been investigated for tracking organ surfaces in laparoscopic images [5], [24], [51]. Our tracking system is based on [4]. This method uses the Speeded-up robust features detector (SURF) and the Lucas-Kanade (LK) optical flow [58] for the tracking stage. This combination has been proven to be suitable for motion tracking in laparoscopic images.

\subsection{Liver Soft-tissue Modeling}

The used pre-operative data consist of a set of CT-scans preoperatively performed on a patient. We exploit these data to build the anatomical model of the liver. The liver model is built following three steps: Segmentation and mesh generation, Volumetric model generation (capable of computing FEM) and tissue parametrization.

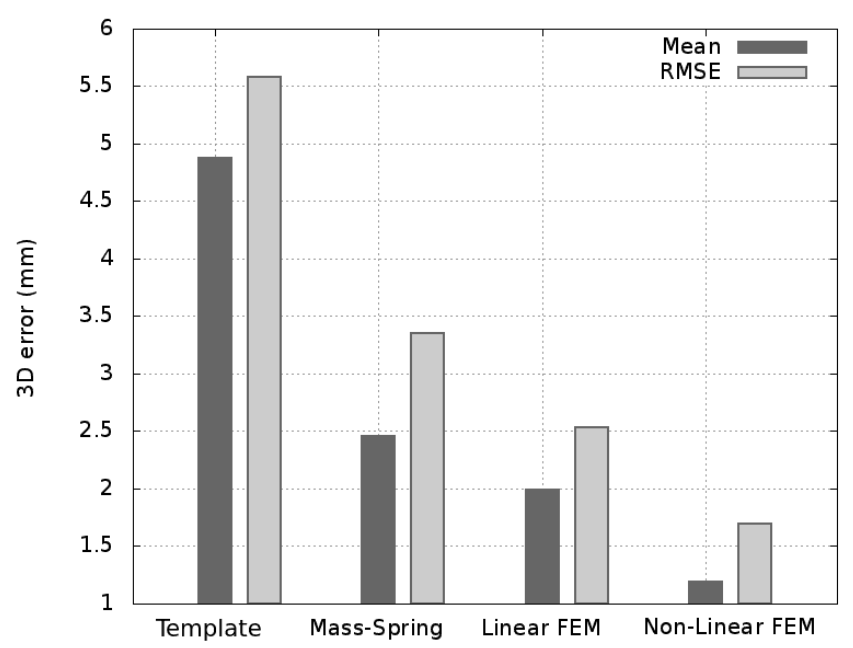

(a)

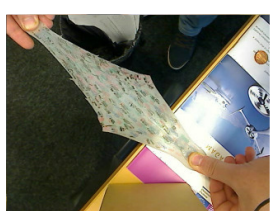

Last Frame

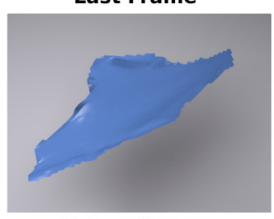

Ground Truth
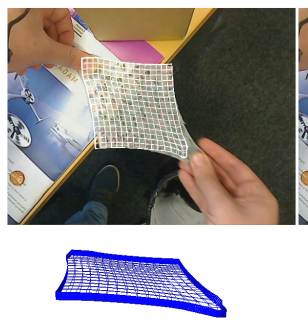

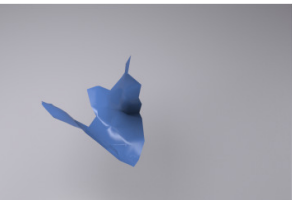

Template-based

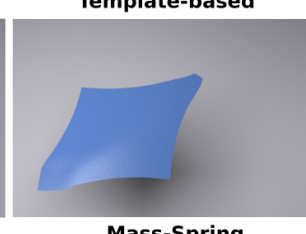

(b)

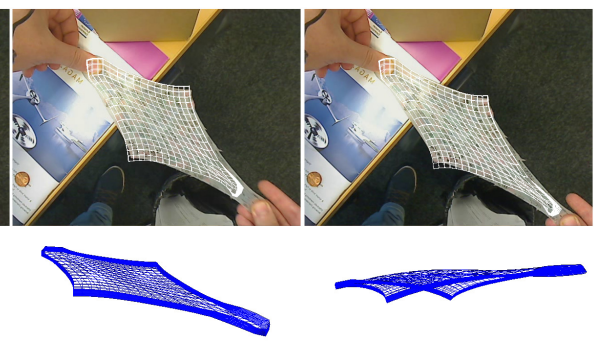

(c)
Fig. 12. 3D shape recovery of a silicone-like material deformation: Our method produces the lowest error in comparison with other methods on deformation 4.

Segmentation: Although this study does not address the challenging task of surface reconstruction from CT-scan images, a good surface representation is very important for physical simulation operations. We use a segmentation technique to generate liver surface from the volumetric CT images. The segmentation is done semi-manually using active contour technique (Snakes) [11] available in the software itksnap [59]. Usually, a mesh-smoothing step is required after segmentation to reduce the number of triangles and obtain a suitable mesh for the volumetric mesh generation.

Volumetric mesh generation: Volumetric meshes are necessary for a finite element modeling. Thus, from the previously generated surface we build a volume representation of the liver composed of linear P1 tetrahedral elements, using 3D Delaunay 
triangulation algorithm which is available in CGal library [47]. The number and type of elements are to be chosen carefully in order to ensure real-time performance as well as sufficient accuracy. In our case, the used model is composed of 3391 linear P1 tetrahedral elements.

Parametrisation: Several studies have measured the characteristics of the liver soft-tissue, using different measurement devices on living and non-living tissues []. In this study, the elastic parameters are set according to [60] to Young's modulus $E=27 \mathrm{KPa}$ and Poisson Coefficient $v=0.45$.

These three steps are commonly performed before a surgical procedure which permit to our framework to easily take advantages of the data. The Young's modulus and Poisson Coefficient are extracted from a textbook and do not suit exactly the considered liver.

The results illustrated in Figure 13 report a visually correct 3D elastic augmentation of the liver model on the laparoscopic image with a good 3D shape recovery.
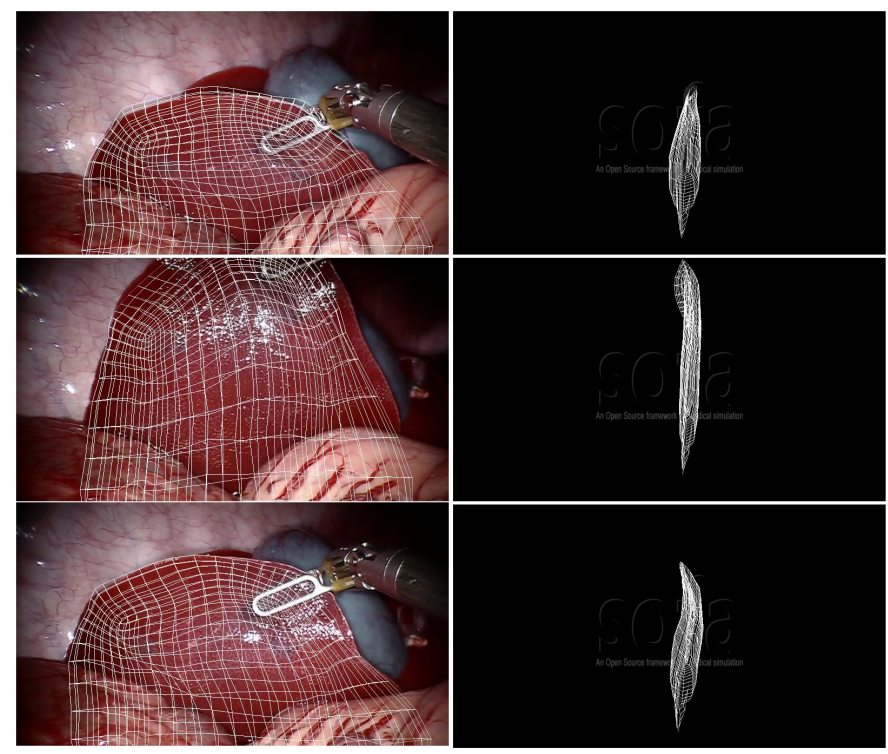

Fig. 13. 3D elastic augmentation of the mechanical model on the laparoscopic images acquired form a monocular camera. The augmentation is effective (left) even when the deformation generated by the instrument forces the lobe of the liver to fold (right).

\section{Self-occluded SuRface}

We tested our method on two video sequences with self-occluded surfaces. We also perform comparison with the elastic method without self-occlusion handling, and the isometric approach [56] where we compare the $3 \mathrm{D}$ reconstruction error with respect to the ground truth by computing the mean and RMS error.

Deformation 5 involves a surface with an extensibility of $10 \%$ and an occluded region of $25 \%$ where deformation 6 is done on a surface with an extensibility of $30 \%$ and an occluded region of $60 \%$ (see Figure 14). The SURF are tracked over frames using a classical optical flow algorithm which is known to be prone to drifting. Thereby, we apply a very strict threshold on the detector to reduce the possible cases of drifting. Once the initial set of features is extracted we apply the condition of Eq. 21 with a value $\tau=10 \mathrm{~mm}$. The features lying on the occluded region will nevertheless remain subject to positioning error. However, the equal distribution of the stretching forces permit to minimize their impact on the recovery of the global shape.

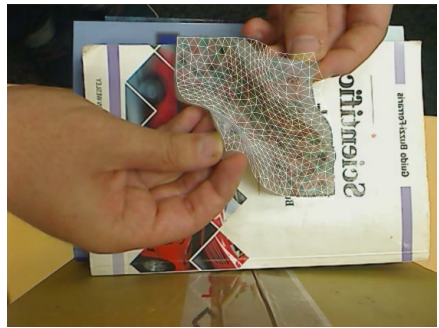

(a)

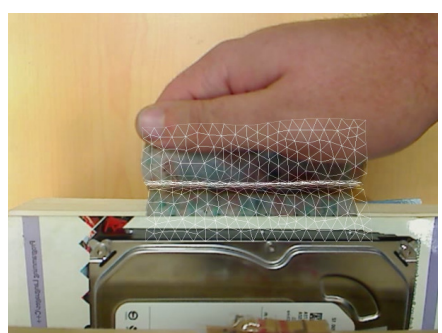

(c)

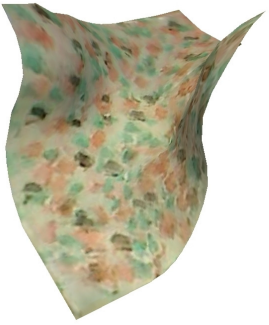

(b)

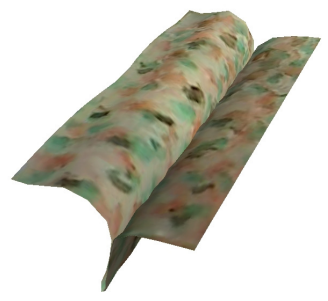

(d)
Fig. 14. Monocular 3D reconstruction of self-occluded elastic surfaces with (a) and (c) the camera view of the registered mesh and (b) and (d) the recovered 3D shape. (top: Deformation 5, bottom: Deformation 6)

The results illustrated in Figure 15 show that in both experiments our method produces a visually correct 3D shape with the lowest 3D error in comparison to other approaches that fail in recovering the 3D surface. We report for our method when using the constraints of Eq. 21, a mean error of $0.51 \mathrm{~mm}$ and a RMS error of $0.66 \mathrm{~mm}$ for deformation 5 and a mean error of $1.72 \mathrm{~mm}$ and RMS error of $2.25 \mathrm{~mm}$ for deformation 6 .

\section{Limitations AND Discussion}

The experiments conducted in this paper involve synthetic materials (where mechanical properties are known) and a relatively simple experimental protocol where the camera is fixed and the scene is almost limited to the deformable object we want to estimate. Since these preliminary results exhibit the relevance of our approach, it allows to consider more complex applications such as augmented reality on inflatable materials or more complex surgical scenarios, where many occlusions happen while the surgeon is manipulating soft anatomical structures with surgical tools. However, the presented method is only valid for a certain range of deformations and cannot yet compete with other $3 \mathrm{D}$ reconstruction techniques that use stereoscopy, moving scope approaches or shape-from-X [61]. Adapting the current approach may include more advanced mechanical models which consider anisotropy and heterogeneity [7]. The ability to compute organs 3D meshes and to retrieve mechanical parameters in an offline stage (form CTscans in case of surgery), makes the overall framework suitable for real like scenarios. Moreover, while the experiments highlight the importance of the boundary conditions, it remains difficult to process them from the pre-operative images. Our work can take 


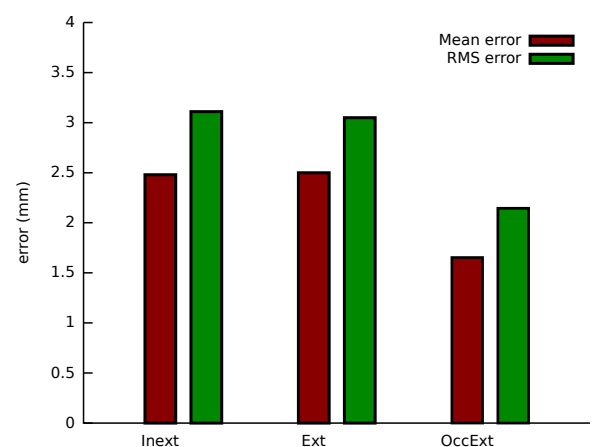

(a) Deformation 5: Comparison

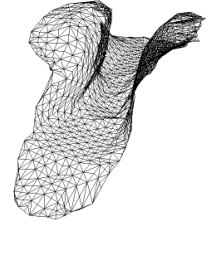

(b) Ground truth

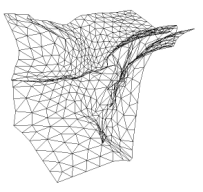

(f) Without SOC

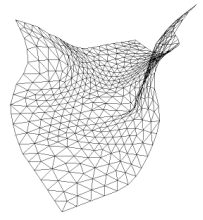

(g) With SOC

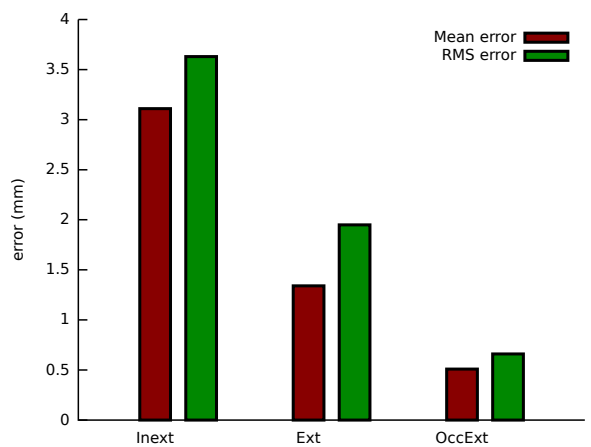

(c) Deformation 6: Comparison

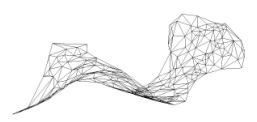

(h) Isometric

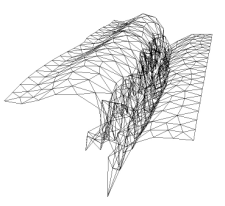

(i) Without SOC

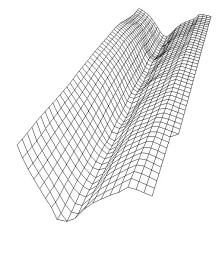

(d) Ground truth

(e) Isometric

Fig. 15. Experiments conducted on the silicone dataset with self-occluded regions. (a) and (c) show the 3D error computed between the recovered mesh and the ground truth using the isometric method, our method without and with self-occlusion constraints (SOC); with the experiment 5 and 6 respectively. (b) and (d) show the ground truth. (e), (f), (g), (h), (i) and (j) show the 3D recovered mesh using each technique.

advantage of recent atlas-based approaches [62] in order to transfer these boundary constraints.

\section{Conclusion}

We have proposed in this paper a real time and efficient method to capture and augment highly elastic objects from a single view. This method makes use of a mechanical model of the deformable object in the context of non linear elasticity. With respect to many existing approaches, this method makes it possible to avoid the definition of ad-hoc constraints to solve the ambiguities of reconstruction. The experiments conducted in this paper prove that the method is flexible in the sense that a classical model the St Venant-Kirchhoff model- has been proven to be sufficient to handle various applications with a good accuracy. These experiments also highlight the importance of the correct definition of boundary conditions to obtain a system that is sufficiently constrained to yield good results and convergence. Moreover, we propose a homogenization constraint that allows to manage, in an elegant manner, considerable self-occlusions. This technique, tested on real data gives visually correct mesh recovery with low registration error. The integration of a fully perspective model in the minimization image term consists one of our future works. An image-based mesh cutting capable to handle volumetric models composed with tetrahedral elements will be integrated in the very near future [63]. This can be of a high interest during minimally invasive surgeries where tumor resection may occur. From a theoretical side of view, an extended study on the impact of boundary conditions on the convergence of the system is obviously important to conduct.

\section{ACKNOWLEDGMENTS}

The authors would like to thank Erwan Kerrien for reliable discussions regarding this work.

\section{REFERENCES}

[1] M. Silcowitz-Hansen, S. Niebe, and K. Erleben, "A nonsmooth nonlinear conjugate gradient method for interactive contact force problems," Vis. Comput., vol. 26, no. 6-8, pp. 893-901, Jun. 2010.

[2] S. Nicolau, L. Soler, D. Mutter, and J. Marescaux, "Augmented reality in laparoscopic surgical oncology," Surgical Oncology, vol. 20, no. 3, pp. $189-201,2011$.

[3] H. Kenngott, M. Wagner, M. Gondan, F. Nickel, M. Nolden, A. Fetzer, J. Weitz, L. Fischer, S. Speidel, H.-P. Meinzer, D. Bckler, M. Bchler, and B. Mller-Stich, "Real-time image guidance in laparoscopic liver surgery: first clinical experience with a guidance system based on intraoperative ct imaging," Surgical Endoscopy, vol. 28, no. 3, pp. 933-940, 2014.

[4] N. Haouchine, J. Dequidt, I. Peterlik, E. Kerrien, M.-O. Berger, and S. Cotin, "Image-guided simulation of heterogeneous tissue deformation for augmented reality during hepatic surgery," in Mixed and Augmented Reality (ISMAR), 2013 IEEE International Symposium on, Oct 2013, pp. 199-208.

[5] P. Pratt, D. Stoyanov, M. Visentini-Scarzanella, and G.-Z. Yang, "Dynamic guidance for robotic surgery using image-constrained biomechanical models," in Medical Image Computing and Computer-Assisted Intervention, 2010, vol. 6361, pp. 77-85.

[6] S. Suwelack, S. Röhl, S. Bodenstedt, D. Reichard, R. Dillmann, T. dos Santos, L. Maier-Hein, M. Wagner, J. Wünscher, H. Kenngott et al., "Physics-based shape matching for intraoperative image guidance," Medical physics, vol. 41, no. 11, p. 111901, 2014.

[7] N. Haouchine, S. Cotin, I. Peterlik, J. Dequidt, M. Lopez, E. Kerrien, and M.-O. Berger, "Impact of soft tissue heterogeneity on augmented reality for liver surgery," Visualization and Computer Graphics, IEEE Transactions on, vol. 21, no. 5, pp. 584-597, May 2015.

[8] M. Sermesant, C. Forest, X. Pennec, H. Delingette, and N. Ayache, "Deformable biomechanical models: Application to 4D cardiac image analysis," Medical Image Analysis, vol. 7, no. 4, pp. 475-488, 2003.

[9] M. Baumann, P. Mozer, V. Daanen, and J. Troccaz, "Prostate biopsy assistance system with gland deformation estimation for enhanced precision," in Proceedings of the 12th International Conference on Medical Image Computing and Computer-Assisted Intervention: Part I, ser. MICCAI '09. Berlin, Heidelberg: Springer-Verlag, 2009, pp. 67-74.

[10] H. Courtecuisse, J. Allard, P. Kerfriden, S. P. Bordas, S. Cotin, and C. Duriez, "Real-time simulation of contact and cutting of heterogeneous soft-tissues," Medical Image Analysis, vol. 18, no. 2, pp. 394 - 410, 2014.

[11] M. Kass, A. Witkin, and D. Terzopoulos, "Snakes: Active contour models," International Journal of Computer Vision, vol. 1, no. 4, pp. $321-331,1988$ 
[12] T. Shen, H. Li, and X. Huang, "Active volume models for medical image segmentation," IEEE Trans. Med. Imaging, vol. 30, no. 3, pp. 774-791, 2011.

[13] J. Schaerer, C. Casta, J. Pousin, and P. Clarysse, "A dynamic elastic model for segmentation and tracking of the heart in mr image sequences," Medical Image Analysis, vol. 14, no. 6, pp. 738 - 749, 2010.

[14] S. Speidel, S. Roehl, S. Suwelack, R. Dillmann, H. Kenngott, and B. Mueller-Stich, "Intraoperative surface reconstruction and biomechanical modeling for soft tissue registration," in Proc. Joint Workshop on New Technologies for Computer Robot Assisted Surgery, 2011.

[15] G. S. P. Miller, "The motion dynamics of snakes and worms," in In Proceedings of ACM SIGGRAPH 1988, 1988, pp. 169-173.

[16] L. Ibai, A. Hugo, A. Iker, and B. Diego, "Real-time deformation, registration and tracking of solids based on physical simulation," in Mixed and Augmented Reality (ISMAR), 2014 IEEE International Symposium on, 2014.

[17] T. F. Cootes, C. J. Taylor, D. H. Cooper, and J. Graham, "Active shape models\&mdash;their training and application," Comput. Vis. Image Underst., vol. 61, no. 1, pp. 38-59, Jan. 1995

[18] I. Matthews and S. Baker, "Active appearance models revisited," Int. J. Comput. Vision, vol. 60, no. 2, pp. 135-164, Nov. 2004.

[19] M. Salzmann, J. Pilet, S. Ilic, and P. Fua, "Surface deformation models for nonrigid 3d shape recovery," IEEE Trans. Pattern Anal. Mach. Intell., vol. 29, no. 8, pp. 1481-1487, Aug. 2007.

[20] F. L. Bookstein, "Principal warps: Thin-plate splines and the decomposition of deformations," IEEE Trans. Pattern Anal. Mach. Intell., vol. 11, no. 6, pp. 567-585, Jun. 1989

[21] T. W. Sederberg and S. R. Parry, "Free-form deformation of solid geometric models," SIGGRAPH Comput. Graph., vol. 20, no. 4, pp. 151160, Aug. 1986.

[22] A. Bartoli, "Maximizing the predictivity of smooth deformable image warps through cross-validation," J. Math. Imaging Vis., vol. 31, no. 2-3, pp. 133-145, Jul. 2008.

[23] J. Lim and M. Yang, "A direct method for modeling non-rigid motion with thin plate spline," in Proceedings of IEEE Conference on Computer Vision and Pattern Recognition (CVPR'05), 12005, pp. 1196-1202.

[24] R. Richa, A. P. L. Bó, and P. Poignet, "Robust 3d visual tracking for robotic-assisted cardiac interventions," in Proceedings of the 13th international conference on Medical image computing and computerassisted intervention, ser. MICCAI2010, 2010, pp. 267-274.

[25] D. Rueckert, L. Sonoda, C. Hayes, D. Hill, M. Leach, and D. Hawkes, "Nonrigid registration using free-form deformations: application to breast $\mathrm{mr}$ images," Medical Imaging, IEEE Transactions on, vol. 18, no. 8, pp. 712-721, Aug 1999.

[26] A. Bartoli and A. Zisserman, "Direct estimation of non-rigid registration," in In British Machine Vision Conference, 2004, pp. 899-908.

[27] D. Pizarro and A. Bartoli, "Feature-based deformable surface detection with self-occlusion reasoning," International Journal of Computer Vision, vol. 97, no. 1, pp. 54-70, 2012.

[28] J. Pilet, V. Lepetit, and P. Fua, "Fast non-rigid surface detection, registration and realistic augmentation," Int. J. Comput. Vision, vol. 76, no. 2, pp. 109-122, Feb. 2008.

[29] J. Zhu and M. R. Lyu, "Progressive finite newton approach to real-time nonrigid surface detection," in Computer Vision and Pattern Recognition, 2007. CVPR'07. IEEE Conference on. IEEE, 2007, pp. 1-8.

[30] V. Gay-Bellile, A. Bartoli, and P. Sayd, "Direct estimation of nonrigid registrations with image-based self-occlusion reasoning," IEEE Trans. Pattern Anal. Mach. Intell., vol. 32, no. 1, pp. 87-104, Jan. 2010.

[31] A. Hilsmann, D. C. Schneider, and P. Eisert, "Realistic cloth augmentation in single view video under occlusions," Comput. Graph., vol. 34, no. 5 , pp. 567-574, Oct. 2010

[32] S. Shen, W. Shi, and Y. Liu, "Monocular 3-d tracking of inextensible deformable surfaces under 12-norm," Trans. Img. Proc., vol. 19, no. 2, pp. 512-521, Feb. 2010.

[33] M. Perriollat, R. Hartley, and A. Bartoli, "Monocular template-based reconstruction of inextensible surfaces," International Journal of Computer Vision, vol. 95, no. 2, pp. 124-137, 2011

[34] M. Salzmann and P. Fua, "Linear local models for monocular reconstruction of deformable surfaces," Pattern Analysis and Machine Intelligence, IEEE Transactions on, vol. 33, no. 5, pp. 931-944, May 2011.

[35] M. Salzmann, R. Hartley, and P. Fua, "Convex optimization for deformable surface 3-d tracking," in Computer Vision, 2007. ICCV 2007. IEEE 11th International Conference on, Oct 2007, pp. 1-8.

[36] F. Moreno-Noguer, M. Salzmann, V. Lepetit, and P. Fua, "Capturing 3d stretchable surfaces from single images in closed form," in Computer Vision and Pattern Recognition, 2009. CVPR 2009. IEEE Conference on, June 2009, pp. 1842-1849.
[37] A. Agudo, B. Calvo, and J. M. M. Montiel, "Finite element based sequential bayesian non-rigid structure from motion," in Computer Vision and Pattern Recognition, 2012. CVPR'12. IEEE Conference on, 2012, pp. $1418-1425$

[38] S. Wuhrer, J. Lang, and C. Shu, "Tracking complete deformable objects with finite elements." in 3DIMPVT. IEEE, 2012, pp. 1-8.

[39] A. Malti, R. Hartley, A. Bartoli, and J.-H. Kim, "Monocular templatebased 3d reconstruction of extensible surfaces with local linear elasticity," in Computer Vision and Pattern Recognition (CVPR), 2013 IEEE Conference on, 2013, pp. 1522-1529.

[40] N. Haouchine, J. Dequidt, M.-O. Berger, and S. Cotin, "Single view augmentation of elastic objects," in Mixed and Augmented Reality (ISMAR), 2014 IEEE International Symposium on, Sep 2014, pp. 199-208.

[41] S. F. Gibson and B. Mirtich, "A survey of deformable modeling in computer graphics," Mitsubishi Electronic Research Laboratory, Tech. Rep., November 1997.

[42] A. Nealen, M. Müller, R. Keiser, E. Boxerman, and M. Carlson, "Physically based deformable models in computer graphics," in Computer Graphics Forum, vol. 25 (4), 2006, pp. 809-836.

[43] E. Sifakis and J. Barbic, "Fem simulation of 3d deformable solids: A practitioner's guide to theory, discretization and model reduction," in ACM SIGGRAPH 2012 Courses, ser. SIGGRAPH '12. New York, NY, USA: ACM, 2012, pp. 20:1-20:50.

[44] J. Barbic and D. L. James, "Real-time subspace integration for st. venantkirchhoff deformable models," in ACM Transactions on Graphics (TOG), vol. 24(3). ACM, 2005, pp. 982-990.

[45] H. Zhong, M. P. Wachowiak, and T. M. Peters, "A real time finite element based tissue simulation method incorporating nonlinear elastic behavior," Computer Methods in Biomechanics and Biomedical Engineering, vol. 8 , no. 3, pp. 177-189, 2005.

[46] R. Kikuuwe, H. Tabuchi, and M. Yamamoto, "An edge-based computationally efficient formulation of saint venant-kirchhoff tetrahedral finite elements," ACM Transactions on Graphics (TOG), vol. 28, no. 1, p. 8 , 2009.

[47] "CGAL, Computational Geometry Algorithms Library," 2015, http://www.cgal.org.

[48] C. Guébert, C. Duriez, and L. Grisoni, "Unified processing of constraints for interactive simulation," in Workshop in Virtual Reality Interactions and Physical Simulation VRIPHYS' 2008, M. T. François Faure, Ed. Grenoble, France: Eurographics association, 2008, pp. 21-29.

[49] A. Shaji, A. Varol, P. Fua, Yashoteja, A. Jain, and S. Chandran, "Resolving occlusion in multiframe reconstruction of deformable surfaces," in Computer Vision and Pattern Recognition Workshops (CVPRW), 2011 IEEE Computer Society Conference on, June 2011, pp. 31-36.

[50] H. Bay, A. Ess, T. Tuytelaars, and L. Van Gool, "Speeded-up robust features (SURF)," Comput. Vis. Image Underst., vol. 110, no. 3, pp. 346359, Jun. 2008.

[51] M. C. Yip, D. G. Lowe, S. E. Salcudean, R. N. Rohling, and C. Y. Nguan, "Tissue tracking and registration for image-guided surgery," Medical Imaging, IEEE Transactions on, vol. 31, no. 11, pp. 2169-2182, Nov 2012.

[52] F. Faure, C. Duriez, H. Delingette, J. Allard, B. Gilles, S. Marchesseau, H. Talbot, H. Courtecuisse, G. Bousquet, I. Peterlik, and S. Cotin, "SOFA: A Multi-Model Framework for Interactive Physical Simulation," in Soft Tissue Biomechanical Modeling for Computer Assisted Surgery, Y. Payan, Ed. Springer, Jun. 2012

[53] J. Bosman, N. Haouchine, J. Dequidt, I. Peterlik, S. Cotin, and C. Duriez, "The role of ligaments: Patient-specific or scenario-specific?" in Biomedical Simulation, ser. Lecture Notes in Computer Science, F. Bello and S. Cotin, Eds. Springer International Publishing, 2014, vol. 8789, pp. 228-232.

[54] K. Miller and J. Lu, "On the prospect of patient-specific biomechanics without patient-specific properties of tissues," Journal of the Mechanical Behavior of Biomedical Materials, vol. 27, no. 0, pp. 154 - 166, 2013.

[55] N. Snavely, S. M. Seitz, and R. Szeliski, "Photo tourism: Exploring photo collections in 3d," in ACM SIGGRAPH 2006. New York, NY, USA: ACM, 2006, pp. 835-846.

[56] A. Bartoli, Y. Gerard, F. Chadebecq, and T. Collins, "On template-based reconstruction from a single view: Analytical solutions and proofs of well-posedness for developable, isometric and conformal surfaces," in Computer Vision and Pattern Recognition (CVPR), 2012 IEEE Conference on, June 2012, pp. 2026-2033.

[57] S. Cotin, H. Delingette, and N. Ayache, "Real-time elastic deformations of soft tissues for surgery simulation," Visualization and Computer Graphics, IEEE Transactions on, vol. 5, no. 1, pp. 62-73, Jan 1999.

[58] B. D. Lucas and T. Kanade, "An iterative image registration technique with an application to stereo vision," in Proceedings of the 7th in- 
ternational joint conference on Artificial intelligence - Volume 2, ser. IJCAI'81. San Francisco, CA, USA: Morgan Kaufmann Publishers Inc., 1981, pp. 674-679.

[59] P. A. Yushkevich, J. Piven, H. Cody Hazlett, R. Gimpel Smith, S. Ho, J. C. Gee, and G. Gerig, "User-guided 3D active contour segmentation of anatomical structures: Significantly improved efficiency and reliability," Neuroimage, vol. 31, no. 3, pp. 1116-1128, 2006.

[60] I. Peterlík, C. Duriez, and S. Cotin, "Modeling and real-time simulation of a vascularized liver tissue," in Medical Image Computing and Computer-Assisted Intervention, 2012, pp. 50-57.

[61] L. Maier-Hein, P. Mountney, A. Bartoli, H. Elhawary, D. Elson, A. Groch A. Kolb, M. Rodrigues, J. Sorger, S. Speidel, and D. Stoyanov, "Optical techniques for $3 \mathrm{~d}$ surface reconstruction in computer-assisted laparoscopic surgery," Medical Image Analysis, vol. 17, pp. 974-996, 2013.

[62] R. Plantefeve, I. Peterlik, H. Courtecuisse, R. Trivisonne, J.-P. Radoux, and S. Cotin, "Atlas-based transfer of boundary conditions for biomechanical simulation," in International Conference on Medical Image Computing and Computer-Assisted Intervention, ser. MICCAI'14, 2014, pp. 33-40.

[63] C. J. Paulus, L. Untereiner, H. Courtecuisse, S. Cotin, and D. Cazier, "Virtual cutting of deformable objects based on efficient topological operations," The Visual Computer, vol. 31, no. 6-8, pp. 831-841, 2015.

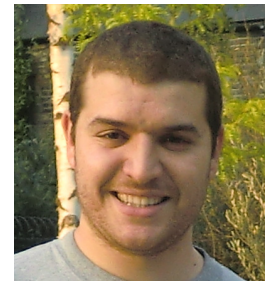

Nazim Haouchine graduated from the University of Science and Technology of Algiers (USTHB), Algeria, in 2010, and received a Masters degree in computer science from the University of Lille 1, Lille, France in 2011. He is now a PhD student at INRIA with Shacra and Magrit groups. His research interests are in augmented reality, computer vision, medical imaging, imageguided simulation for surgery and clinical validations.

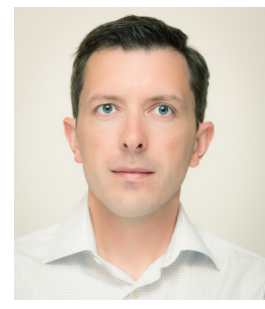

Jérémie Dequidt completed a Ph.D. thesis in Computer Science at the University of Lille 1 in 2005. As a post-doctoral fellow, he joined the SimGroup at CIMIT (Boston, MA) and then INRIA Alcove team, working on interventional radiology simulations. Since september 2008, he is an Assistant Professor in Computer Science at University of Lille 1 . He also is a member of the Inria research-team Shacra. His research interests focus on collision detection and response, mechanical/geometric/adaptive modeling and experimental validations.

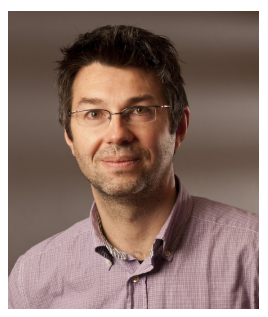

Stephane Cotin joined INRIA in 2007 to hold a position of Research Director. Since January 2010 he is also leader of the SHACRA group, a multidisciplinary team of scientists involved in the field on medical simulation. Before joining INRIA, he was Research Lead for the Medical Simulation Group at CIMIT (Boston, Ma). His main research interests are in physics-based simulation, real-time simulation of soft-tissue deformations, and medical applications of this research.

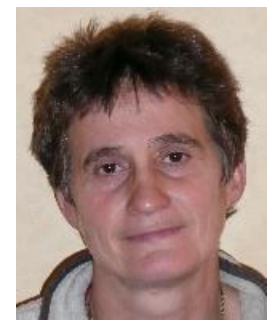

Marie-Odile Berger is presently research scientist at INRIA in computer vision. She is "agregée de mathematiques" and received a PhD in computer science from University Henri Poincaré at Nancy, France. She is currently the head of the computer vision group Magrit at INRIA Nancy Grand est. Her research interests include visual reconstruction, augmented reality, medical imaging, analysis of video sequences. 